\title{
Biological augmentation of rotator cuff repair using bFGF-loaded electrospun poly(lactide-co-glycolide) fibrous membranes
}

This article was published in the following Dove Press journal:

International Journal of Nanomedicine

14 May 2014

Number of times this article has been viewed

\author{
Song Zhao',* \\ Jingwen Zhao ${ }^{3, *}$ \\ Shikui Dong' \\ Xiaoqiao Huangfu' \\ Bin $\mathrm{Li}^{2,3}$ \\ Huilin Yang ${ }^{2,3}$ \\ Jinzhong Zhao' \\ Wenguo $\mathrm{Cui}^{2,3}$ \\ 'Department of Arthroscopic \\ Surgery, Shanghai Jiao Tong University \\ Affiliated Sixth People's Hospital, \\ Shanghai, ${ }^{2}$ Orthopedic Institute, \\ Soochow University, Suzhou, Jiangsu, \\ ${ }^{3}$ Department of Orthopedics, The \\ First Affiliated Hospital of Soochow \\ University, Suzhou, Jiangsu, People's \\ Republic of China \\ *These authors contributed equally \\ to this work
}

Correspondence: Jinzhong Zhao

Department of Arthroscopic Surgery, Shanghai

Jiao Tong University Affiliated Sixth People's Hospital, 600 Yishan Road, Shanghai, 200233, People's Republic of China

Tel +86 21 6436 918| ext 5809।

Email zhaojinzhongdoctor@।63.com

Wenguo Cui

Department of Orthopedics, The First Affiliated Hospital of Soochow University, 188 Shizi St, Suzhou, Jiangsu 215006,

People's Republic of China

Tel +865I2 6778 I420

Email wgcui80@hotmail.com

\begin{abstract}
Clinically, rotator cuff tear (RCT) is among the most common shoulder pathologies. Despite significant advances in surgical techniques, the re-tear rate after rotator cuff (RC) repair remains high. Insufficient healing capacity is likely the main factor for reconstruction failure. This study reports on a basic fibroblast growth factor (bFGF)-loaded electrospun poly(lactideco-glycolide) (PLGA) fibrous membrane for repairing RCT. Implantable biodegradable bFGF-PLGA fibrous membranes were successfully fabricated using emulsion electrospinning technology and then characterized and evaluated with in vitro and in vivo cell proliferation assays and repairs of rat chronic RCTs. Emulsion electrospinning fabricated ultrafine fibers with a core-sheath structure which secured the bioactivity of bFGF in a sustained manner for 3 weeks. Histological observations showed that electrospun fibrous membranes have excellent biocompatibility and biodegradability. At 2, 4, and 8 weeks after in vivo RCT repair surgery, electrospun fibrous membranes significantly increased the area of glycosaminoglycan staining at the tendon-bone interface compared with the control group, and bFGF-PLGA significantly improved collagen organization, as measured by birefringence under polarized light at the healing enthesis compared with the control and PLGA groups. Biomechanical testing showed that the electrospun fibrous membrane groups had a greater ultimate load-to-failure and stiffness than the control group at 4 and 8 weeks. The bFGF-PLGA membranes had the highest ultimate load-to-failure, stiffness, and stress of the healing enthesis, and their superiority compared to PLGA alone was significant. These results demonstrated that electrospun fibrous membranes aid in cell attachment and proliferation, as well as accelerating tendon-bone remodeling, and bFGF-loaded PLGA fibrous membranes have a more pronounced effect on tendon-bone healing. Therefore, augmentation using bFGF-PLGA electrospun fibrous membranes is a promising treatment for RCT.
\end{abstract}

Keywords: rotator cuff tear, bFGF, electrospinning, PLGA, rat model

\section{Introduction}

Rotator cuff tear (RCT) is among the most common shoulder pathologies, leading to debilitating pain, reduced shoulder function, and weakness. Despite significant advances in surgical techniques to optimize the fixation of tendon to bone to achieve the highest possible initial strength, prior studies have reported a relatively high rate of structural failure after rotator cuff (RC) repair. ${ }^{1-5}$ Patient age, tear size and chronicity, tendon quality, muscle atrophy and degeneration, and biologic healing response are considered important risk factors for re-tear. ${ }^{6}$ These risk factors are linked with insufficient healing capacity in these degenerative lesions, which is likely the main factor for failure of reconstruction in RC surgery and is therefore of increasing interest. 
There have recently been remarkable developments in biological approaches to the mechanical reinforcement of RC repair and to the stimulation and enhancement of patients' intrinsic healing potential. Several studies delivered growth factors locally to the tendon repair site via coated sutures or dissolved in a fibrin sealant. ${ }^{7,8}$ Although such augmentations appear promising, growth factors are short-lived and cannot be active for long periods. Gene therapy offers another approach for accurate delivery of the growth factor of interest, ${ }^{9}$ but safety concerns exist with regard to the use of viral vectors. Scaffolds have proved to be safe and can localize growth factors to the repair site. Furthermore, some bioscaffolds may even have the capacity to strengthen RC repair. Combination therapies using appropriate scaffolds loaded with growth factors may prove to be more effective for RC therapy.

Basic fibroblast growth factor (bFGF) is a very potent mitogen and has been shown in vitro to induce fibroblast collagenase production and capillary endothelial cell proliferation, which are required for neovascularization. ${ }^{710,11} \mathrm{After}$ RC injury, bFGF is expressed by fibroblasts and inflammatory cells at the site of tendon repair and is associated with cell proliferation, cell migration, collagen production, and angiogenesis. ${ }^{10-13}$ Various models have suggested that the addition of bFGF may increase the strength of tendon healing and accelerate tendon-bone remodeling. ${ }^{711,14-16}$ Ide et al demonstrated that specimens treated locally with bFGF had an early improvement in bone ingrowth at the tendon-bone interface and biomechanical strength at 2 weeks after RC repair in rats. ${ }^{7}$ Considering the transient effect of bFGF delivery alone, Ide et al additionally implanted acellular dermal matrix scaffolds for RC repair in their later study. ${ }^{17}$ These scaffolds produced a more delayed effect, with a significant increase in enthesis strength and tendon maturity at 6 and 12 weeks postoperatively. ${ }^{17}$ However, these scaffolds, which are derived from dermis, appear to undergo remodeling slowly and may be incorporated by the host to some extent rather than replaced completely. ${ }^{18}$ Additionally, a limited number of studies demonstrated meaningful improvement by combination therapies in chronic RCT models, which mostly reproduce the pathology seen in human RCTs.

Recently, various physical scaffolds have been used to repair RCTs. Specifically, electrospun fibrous scaffolds are effective for tissue separation and drug delivery because of their high aspect ratio, high porosity, and very small pore size. ${ }^{19,20}$ Of these electrospun fibrous membranes, poly(lactide-co-glycolide) (PLGA) is a widely used biomedical polymer because of its biodegradability and biocompatibility. ${ }^{21}$ PLGA and its derivatives have been used in the preparation of membranes by electrospinning and solvent casting film and have shown preliminary effects in RC repair. ${ }^{22}$ Furthermore, electrospun PLGA derivatives can enable bFGF release over 1-2 weeks through blending, emulsion, coaxial, or pre-formulated electrospinning. ${ }^{22}$ With the protection of the electrospun fibers in such systems, bFGFs are able to maintain their activity.

While the size scale and topography of electrospun nanofibers can aid in cell attachment and proliferation, electrospun fibrous mats appear to be one of the most suitable materials for regeneration after RCTs, allowing gaseous and fluid exchanges, absorbing excess exudates, and guiding tissue regeneration. The purpose of this study was to evaluate the extent to which augmentation of chronic RCTs repairs with bFGF-loaded electrospun PLGA fibrous membranes would improve functional and biomechanical outcomes in a rat model (Figure 1). Our hypothesis was that, compared with repairs without augmentation, augmented repairs would aid reconstruction of the tendon-bone insertion and enhance tendon-bone healing in vivo. Emulsion electrospinning was adopted to prepare bFGF-loaded PLGA electrospun fibrous membranes with a core-sheath structure, which was essential for the ability of electrospun fibers to increase the encapsulation efficiency, retard the initial burst release, and maintain bFGF bioactivity. The bFGF-PLGA fibrous membranes were then characterized and evaluated by in vitro and in vivo results of cell proliferation and repairs of RCTs.

\section{Materials and methods Materials}

Poly(lactide-co-glycolide) (PLGA, 90/10, Mw=100 kDa, Mw/ $\mathrm{Mn}=1.83$ ) was prepared by bulk ring-opening polymerization of lactide and glycolide using stannous chloride as an initiator (Jinan Daigang Biomaterial Co, Ltd, Jinan, People's Republic of China). Dichloromethane and trichloromethane were

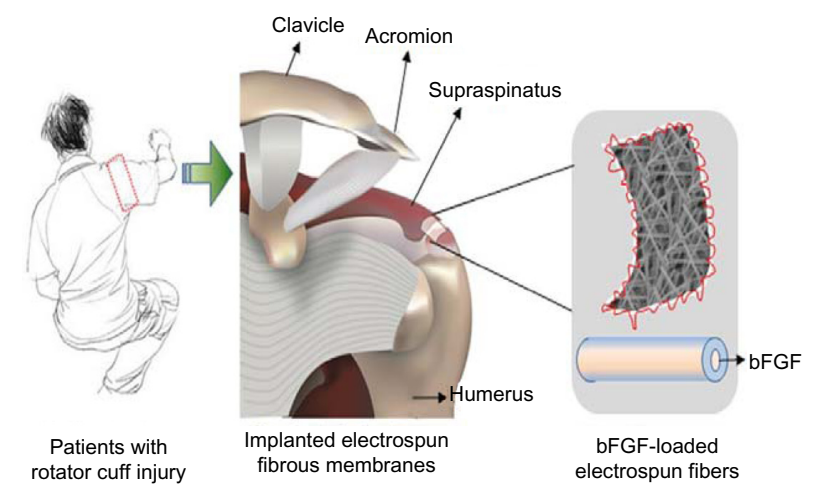

Figure I Augmentation of RCT repair with bFGF-loaded electrospun fibrous membranes.

Abbreviations: bFGF, basic fibroblast growth factor; RCT, rotator cuff tear. 
purchased from Chinese Medicine Group Chemical Reagent Corporation (AR Shanghai, People's Republic of China); $\alpha$-cyclodextrin (suitable for cell culture, $\geq 98 \%$ ) was supplied by Sigma-Aldrich Co (St Louis, MO, USA); and bFGF and the bFGF enzyme-linked immunosorbent assay (ELISA) kit were acquired from R\&D Company (Minneapolis, MN, USA). All other chemicals and solvents were of reagent grade and purchased from Guoyao Regents Company (Shanghai, People's Republic of China).

\section{Fabrication and bFGF-loaded PLGA fibrous membranes}

bFGF $(100 \mu \mathrm{L}, 20 \mu \mathrm{g} / \mathrm{mL})$ was dissolved in $0.6 \mathrm{~mL}$ phosphate-buffered saline (PBS, $50 \mathrm{mM}, \mathrm{pH}$ 7.4) containing $76 \mathrm{mg} / \mathrm{mL}$ cyclodextrin, and the $0.7 \mathrm{~mL}$ solution was dropped into a PLGA/chloroform solution of $12.5 \%$ (w/v, $1 \mathrm{~g}$ PLGA and $7 \mathrm{~mL}$ chloroform), followed by ultrasonication in an ice bath (VC 505; Sonics and Materials Inc., Newtown, CT, USA). The uniform microemulsion solution was fabricated for electrospinning, and the electrospinning technology was as described elsewhere. ${ }^{23}$ Briefly, the electrospinning apparatus was equipped with a high voltage station (Tianjin High Voltage Power Supply Co, Tianjin, People's Republic of China), and the electrospinning voltage was set at $10-25 \mathrm{kV}$. The emulsion was added from a $5 \mathrm{~mL}$ syringe attached to a metal capillary with an exit orifice diameter of $0.6 \mathrm{~mm}$, and the flow rate of $3 \mathrm{~mL} /$ hour was controlled by a microinjection pump (Lange Medical Instrument Co, Baoding, Hebei, People's Republic of China). For the electrospun PLGA solution, $1 \mathrm{~g}$ PLGA was dissolved in $4 \mathrm{~g}$ dichloromethane (DCM) and $2 \mathrm{~g}$ N,N-dimethylformamide (DMF). All the fibrous mats were lyophilized overnight to remove any solvent and water residues and were stored at $4^{\circ} \mathrm{C}$ in a desiccator for further use.

\section{Characterization of fibrous membranes}

Morphology was observed by scanning electron microscopy (SEM; Quanta 200; FEI, Eindhoven, the Netherlands).

Each specimen was examined for the structure of PLGA fibers with a transmission electron microscope (TEM; JEM2100F; JOEL, Tokyo, Japan).

The water contact angles of different fibrous membranes were measured with a KRÜSS GmbH DSA 100 Mk 2 goniometer (Hamburg, Germany) followed by image processing of sessile drop with DSA 1.8 software (Krüss, Hamburg, Germany).

To test the mechanical properties, the dry fibrous membranes were punched into small strips $(70.0 \times 7.0 \times 0.6 \mathrm{~mm})$. Uniaxial tensile tests were performed using an all-purpose mechanical testing machine (5567; Instron, Norwood, MA, USA). The stress-strain curves of the fibrous membranes were constructed from the load deformation curves recorded at a stretching speed of $0.5 \mathrm{~mm} / \mathrm{s}(\mathrm{n}=3)$. Young's modulus, tensile strength, and elongation at break of the fibrous membranes were derived from the stress-strain curves.

\section{BFGF encapsulation}

The emulsion electrospun bFGF-loaded PLGA membrane was dissolved in dichloromethane. The suspension was then centrifuged at 12,000 rpm for 5 minutes using an Anker TGL-16C centrifuge (Hyderabad, India) to remove the upper clear liquid. After evaporating the residual dichloromethane, $1 \mathrm{~mL}$ of PBS (pH 7.4) was added and the solution was tested using the bFGF ELISA assay kit to determine the amount of bFGF. Encapsulation efficiency $(\%)=(\mathrm{P} / \mathrm{Pt}) \times 100$, where $\mathrm{P}$ is the actual protein encapsulated into the membranes and $\mathrm{Pt}$ is the theoretical amount of protein encapsulated into the membranes. ${ }^{24}$

\section{In vitro bFGF release profiles}

We used PBS ( $\mathrm{pH} 7.4$ ) to simulate the physiological environment of the human body for the in vitro release test. An electrospun fibrous membrane was put in the release bottle with $1 \mathrm{~mL}$ PBS and then incubated in an air rotator at $150 \mathrm{rpm}$ and $37^{\circ} \mathrm{C}$. At predetermined time intervals, the supernatant was collected and fresh medium of equal volume was added. The bFGF content of releasing solution was measured using the bFGF ELISA assay kit. ${ }^{23}$

\section{In vitro cell culture on scaffolds}

Human dermal fibroblasts (HDFs), which were used to detect the cell behavior on the electrospun fibrous scaffolds, were isolated from human hypertrophic scars provided by the Department of Plastic and Reconstructive Surgery, Ninth People's Hospital Affiliated to Medical School of Shanghai Jiao Tong University (Shanghai, People's Republic of China). The cells were cultured in Dulbecco's Modified Eagle's medium (DMEM) supplemented with 10\% fetal bovine serum (FBS), penicillin (100 units/mL), and streptomycin (100 $\mu \mathrm{g} / \mathrm{mL})$ (Sigma-Aldrich) in a humidified incubator at $37^{\circ} \mathrm{C}$ with $5 \% \mathrm{CO}_{2}$. Culture medium was replaced every 3 days. Once the HDFs reached $70 \%$ confluence, they were passaged at a ratio of $1: 3$. The second to fourth passages of HDFs were used in this study. ${ }^{21}$

Sterilized electrospun fibrous scaffolds cut into disks with a $5 \mathrm{~mm}$ diameter were pre-wetted with cell culture medium for 2 hours. HDF suspensions $\left(50 \mu \mathrm{L}, 3 \times 10^{4}\right.$ cells $\left./ \mathrm{mL}\right)$ were seeded on the membrane surfaces and placed in 96-well 
culture plates (Costar; Corning Inc., Corning, NY, USA). Cell-seeded scaffolds were then incubated at $37^{\circ} \mathrm{C}$ with $5 \% \mathrm{CO}_{2}$ for 4 hours before $100 \mu \mathrm{L}$ of culture medium was added into each well. The culture medium was changed every second day.

SEM micrographs were taken when HDFs attached and proliferated separately on membranes at 1,3, and 5 days after seeding. Scaffolds were harvested, washed with PBS solution twice, and fixed with $4 \%$ glutaraldehyde for 2 hours at $4{ }^{\circ} \mathrm{C}$. The samples were dehydrated through a series of graded ethanol solutions, followed by rinsing with distilled water three times. Dry specimens were sputter-coated with gold and observed by SEM.

HDF proliferation was investigated using the Cell Counting Kit-8 (CCK-8; Dojindo Molecular Technologies, Inc., Kumamoto, Japan) after incubation for 1, 3, or 5 days. Briefly, the culture medium was removed, and cell-scaffold samples were washed twice with PBS. Subsequently, $100 \mu \mathrm{L}$ culture medium and $10 \mu \mathrm{L} \mathrm{CCK}-8$ reagent were added to each sample and incubated at $37^{\circ} \mathrm{C}$ for 2.5 hours, according to the reagent instructions, in a humidified incubator at $37^{\circ} \mathrm{C}$ with $5 \% \mathrm{CO}_{2}$. Then, $100 \mu \mathrm{L}$ of incubated medium was transferred to a 96-well culture plate, and the absorbance was measured at $450 \mathrm{~nm}$ with a microplate reader (Thermo Labsystems, Waltham, MA, USA). All experiments were performed in triplicate.

\section{Chronic RCT model}

This study used 144 male Sprague-Dawley rats (obtained at 350-400 grams). The surgical procedure was performed according to previously published protocols. ${ }^{25-27}$ At primary surgery, under general anesthesia, the left shoulder was manipulated to completely detach the supraspinatus tendon from its insertion site on the humerus. After fixing the rat in the lateral decubitus position, the deltoid muscle was exposed with a longitudinal incision on the anterolateral aspect of the shoulder and subsequently split. Under adduction, retroversion, and slight internal rotation the supraspinatus insertion on the greater tuberosity of the proximal humerus was revealed. The supraspinatus tendon was marked with a 5-0 PROLENE $^{\circledR}$ suture (Ethicon, Cincinnati, OH, USA) approximately $3 \mathrm{~mm}$ medial from the insertion and detached sharply from its insertion on the greater tuberosity. Finally, the adhesions to the anterior and posterior tissue were released from the supraspinatus tendon. To facilitate the localization of the tendon stump at the time of repair, the suture was knotted with three simple knots. The overlying deltoid muscle and skin was then closed, and the rat was allowed normal cage activity. The rats received weight-adapted pain medication (oral metamizole and subcutaneous buprenorphine) during the postoperative period.

At 3 weeks postoperatively, the animals were randomized into one of three groups. In the control group, the tendon was restored to its anatomic footprint (transosseous repair) ( $\mathrm{n}=48)$. In the experimental groups, 96 rats underwent transosseous repair and were implanted with either pure PLGA membranes $(n=48)$ or bFGF-PLGA membranes $(n=48)$ to augment the repair. The previous scar was re-incised for repair of the chronic degenerated tendon. Under humeroacromial extension, the marking suture was retrieved and the tendon was mobilized. The footprint was decorticated gently with a \#15-blade knife to ensure complete debridement of the native enthesis. Crossed bone tunnels were drilled at the anterior and posterior margins of the footprint using a 22-gauge needle. Suture ends from the tendon were then passed through the bone tunnels and firmly tied over the humeral metaphyseal cortex, anatomically restoring the supraspinatus tendon to its native footprint. In the control group, no further reinforcement was performed. In the experimental groups, the repair was additionally overlapped with PLGA or bFGF-PLGA fibrous membrane, creating a layered construct consisting of membrane and tendon. The subsequent wound closure and postoperative treatment was performed according to the first operation.

\section{Histomorphometric analysis}

Twenty-four animals (eight animals per group per time period) were euthanized at 2, 4, and 8 weeks after surgery and allocated for histomorphometric analysis. After necropsy, the tissue specimens were fixed in 10\% neutral buffered formalin, decalcified with Immunocal (Decal, Congers, NY, USA), and embedded in paraffin. Five-micrometer-thick sections were cut in the coronal plane through the repaired supraspinatus tendon and the greater tuberosity. The tissue sections were stained with hematoxylin and eosin (H\&E), Safranin O/Fast Green, and Picro Sirius red.

Tissue sections stained with Safranin O/Fast Green were analyzed to determine the total area of new fibrocartilage formation at the insertion site of the $\mathrm{RC}$ repair using light microscopy (DM4000B; Leica, Solms, Germany). Digital images of the stained tissue sections were taken using a DFC420C camera (Leica). Image J software (National Institutes of Health, Bethesda, MA, USA) was used to determine the area of new fibrocartilage formation by manually outlining the area of metachromasia on the Safranin O slides 
at a total magnification of $50 \times$. The total area of metachromasia was then recorded in $\mathrm{mm}^{2}$ for each specimen. ${ }^{28}$

Tissue sections were stained with Picro Sirius red for semiquantitative analysis of collagen deposition and maturation at the repair site. By quantifying the collagen birefringence under polarized light microscopy (Eclipse E800; Nikon, Melville, NY, USA), differences in collagen maturity and organization in the healing tendon could be detected. Measurements were obtained by rotating the polarization plane until maximum brightness was obtained. To facilitate comparisons between groups, tissues were embedded and cut to a uniform thickness, and the light intensities were measured under exactly the same conditions of illumination, and during the same sitting, for all specimens. After a photomicrograph was captured, the image was imported into Image $\mathrm{J}$, in which the image underwent 8-bit digitization, producing an image in which noncollagenous material was dark (zero) and collagenous material was depicted in grayscale with values from 1 to 255 . Ten areas $(50 \times 50 \mu \mathrm{m})$ were randomly selected from the tendon region adjacent to the insertion site and the grayscale values were measured and recorded. ${ }^{28}$

\section{Biomechanical testing}

Twenty-four animals were allocated for biomechanical testing (eight rats per group per time point) at 2, 4, or 8 weeks after surgery. After being thawed at room temperature, the humerus with attached supraspinatus tendon was meticulously dissected from the surrounding tissues. The supraspinatus muscle belly was removed from the tendon. The scar tissue was also removed. A digital caliper was used to measure the cross-sectional area of each supraspinatus tendon at the point of insertion into the humerus. The specimen was then transferred to a custom-designed uniaxial testing system. The tendon was secured in a screw grip using sandpaper and ethyl cyanoacrylate. Fracture through the humeral physis was prevented by placing the humerus in a vice grip. The supraspinatus tendon was secured to a $45 \mathrm{~N}$ load cell attached to a linear bearing that allowed alignment of the tendon in the direction of its pull. The humeral jig was secured to the linear stage, and grip-to-grip distance was standardized across all specimens. The specimen was preloaded to $0.1 \mathrm{~N}$ and then loaded to failure at a rate of $14 \mu \mathrm{m} / \mathrm{s}$, corresponding to approximately $0.4 \%$ strain. The ultimate load-to-failure and the failure site were recorded. Displacement was measured using a $1 \mu \mathrm{m}$ resolution micrometer system attached to the linear stage. The linear region of the load-displacement curve was used to calculate the stiffness for each specimen.
Ultimate stress at failure was calculated by dividing the ultimate load-to-failure by the cross-sectional area. This testing protocol has been used in previous studies. ${ }^{28-30}$

\section{Data analysis}

Approval for this study was obtained from the University Animal Care Committee of Shanghai Jiao Tong University. All authors were blinded to which study group or time interval the specimens belonged to at the time of histomorphometric analysis and biomechanical testing.

All data are presented as the mean \pm standard deviation (SD). Statistical analysis was performed using one-way analysis of variance (ANOVA) with post hoc testing using the Holm-Šidák method. Significance was set at $P<0.05$.

\section{Results \\ Fiber characterization}

PLGA and bFGF-loaded PLGA fibers (bFGF-PLGA) were successfully fabricated through the emulsion electrospinning method. The electrospun fibrous morphology of electrospun PLGA and bFGF-PLGA fibrous membranes are shown in Figure $2 \mathrm{~A}$ and $\mathrm{B}$. There were no beads in the fibrous structure, and the fibers were uniform in size, forming randomly interconnected structures that appeared smooth in all samples. The TEM micrographs of the fibers obtained are shown in Figure $2 \mathrm{C}$ and $\mathrm{D}$. The core phase of bFGF and shell phase of PLGA can be seen in the emulsion electrospun bFGF-PLGA fibers (Figure 2D) while the uniform phase of PLGA can be clearly observed in the electrospun PLGA fibers (Figure 2C).

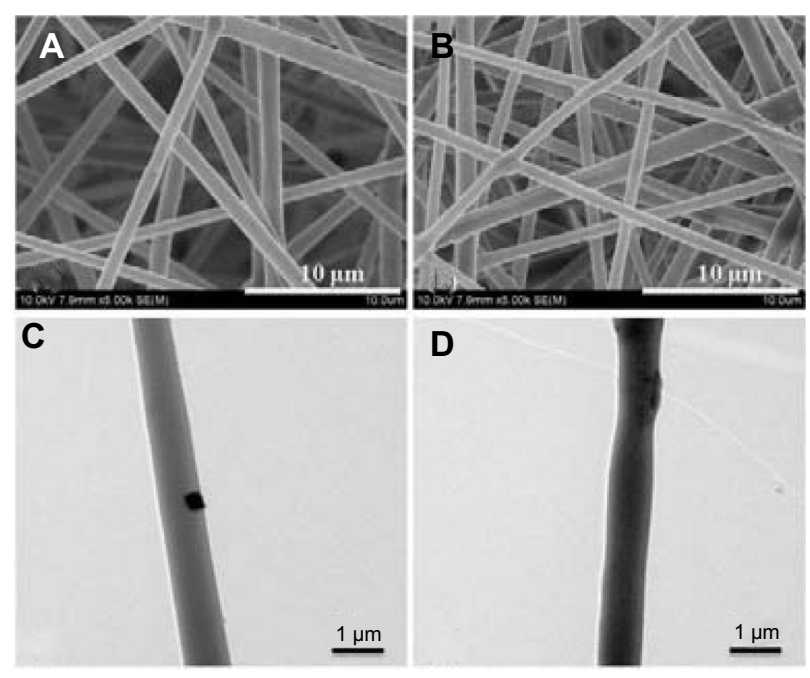

Figure 2 SEM images of electrospun PLGA (A) and bFGF-PLGA (B); TEM images of electrospun PLGA (C) and bFGF-PLGA (D) fibers.

Abbreviations: bFGF, basic fibroblast growth factor; PLGA, poly(lactide-coglycolide); SEM, scanning electron microscopy; TEM, transmission electron microscopy. 
Fiber diameters were $1.04 \pm 0.25 \mu \mathrm{m}$ and $0.92 \pm 0.26 \mu \mathrm{m}$ for electrospun PLGA and bFGF-PLGA fibrous membranes, respectively.

To clarify the effects of the emulsion electrospinning methods on the surface properties of electrospun fibers, water contact angles of electrospun fibrous membranes were measured. The water contact angles were $126.3^{\circ} \pm 3.7^{\circ}$ and $123.2^{\circ} \pm 4.1^{\circ}$ for electrospun PLGA and bFGF-PLGA fibrous membranes, respectively.

Stress-strain measurements were conducted to clarify the mechanical properties of bFGF-loaded electrospun membranes. The tensile strengths of PLGA and bFGF-PLGA fibrous membranes were $3.33 \pm 0.31 \mathrm{MPa}$ and $3.18 \pm 0.29 \mathrm{MPa}$, respectively, while the tensile moduli were $46.14 \pm 4.42 \mathrm{MPa}$ and 39.36 $\pm 3.69 \mathrm{MPa}$, respectively, and statistical analysis indicates no significant difference between the PLGA and bFGF-loaded PLGA fibrous membranes $(P>0.05)$.

\section{BFGF encapsulation}

In the emulsion electrospinning, cyclodextrin was chosen for inclusion in the inner aqueous phase, increasing the viscosity of the internal aqueous phase and stabilizing the emulsion, in which it acts as a stabilizing excipient for bFGF and results in high encapsulation efficiency. The protein encapsulation efficiency of the bFGF-PLGA fibrous membrane was $36.26 \% \pm 7.61 \%$, and the amount of bFGF loaded in electrospun fibers was $0.7252 \times 10^{-3} \pm 0.07 \%(\mathrm{w} / \mathrm{w})$.

\section{In vitro bFGF release profiles}

The cumulative release of bFGF from core-sheath structured bFGF-PLGA electrospun fibrous membrane is shown in Figure 3. A burst release of $15.4 \%$ was detected during the

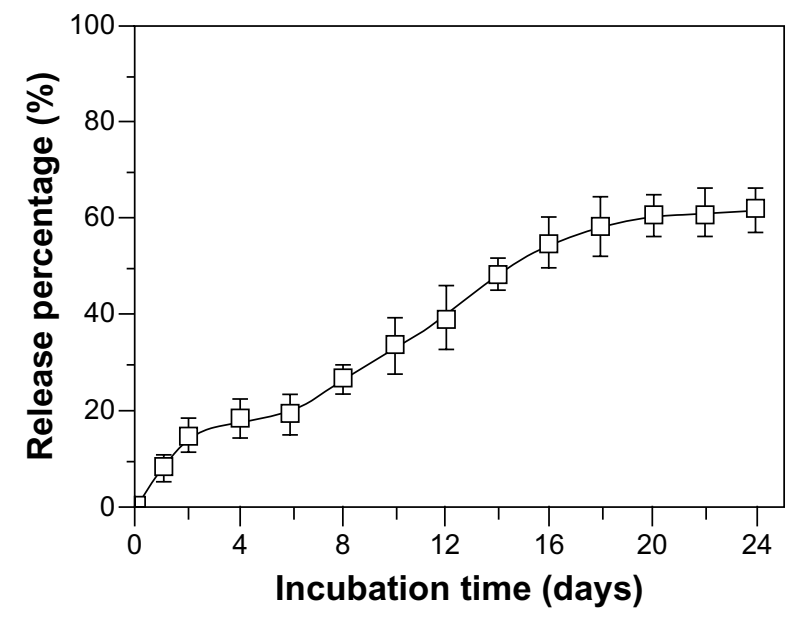

Figure 3 In vitro bFGF release profiles of bFGF-PLGA fibrous membranes. Abbreviations: bFGF, basic fibroblast growth factor; PLGA, poly(lactide-coglycolide). initial 2 days, followed by a constant release for 14 days (days 2-16) and then a slow release for another 8 days (days 16-24). bFGF located on or near the fiber surface results in the burst release, and the encapsulation of bFGF into the fiber core can apparently alleviate the initial burst release. The cumulative releasing percentage of bFGF in the bFGF-PLGA fibers was $62.2 \%$, indicating that the activity of bFGF was well-protected in core-shell fibers during the incubation time.

\section{Loading bFGF for promoting fibroblasts cells}

The proliferation of HDF cells on the surface of the tissue culture plate, electrospun PLGA, and bFGF-PLGA fibrous membranes were compared after 1, 3, and 5 days (Figure 4). There was major cell growth on the electrospun bFGF-PLGA fibrous membranes, and the cells were observed to be characteristically spindle-shaped (Figure 4B), while the PLGA membrane surface showed the fewest cells (Figure 4A). The HDF cell number for all the groups increased with increasing time in culture (Figure 4C). Electrospun PLGA fibrous membranes increased slowly, implying that there was low HDF proliferation on the electrospun PLGA fibrous membranes. However, there were statistically significant differences $(P<0.05)$ for bFGF-PLGA fibrous membranes compared with PLGA fibrous membranes after 3 days and 5 days, which indicated that bFGF in PLGA fibers resulted in further promotion of HDF cell proliferation.

\section{Macroscopic observations}

No gross evidence of infection was observed at the surgical site in any of the specimens, and no adhesions or contractures limited the shoulder range of motion. The repaired tendons were in continuity with the bone in all animals at the time of sacrifice. No qualitative differences in the gross appearance of either the supraspinatus tendon or healing enthesis were appreciated during necropsy. The shoulders in the experimental groups had remnants of the membranes present at the tendon attachment site footprint at 2 and 4 weeks after surgery, while no remnants were observed later.

\section{Histological analysis}

\section{Cellularity and host tissue response}

Under low magnification microscopy, the membranes remaining at 2 and 4 weeks postoperatively in the experimental and bFGF-PLGA groups exhibited a faster degradation velocity. At 8 weeks, the membranes had degraded completely (Figure 5A). At 2 weeks, high magnification showed that 

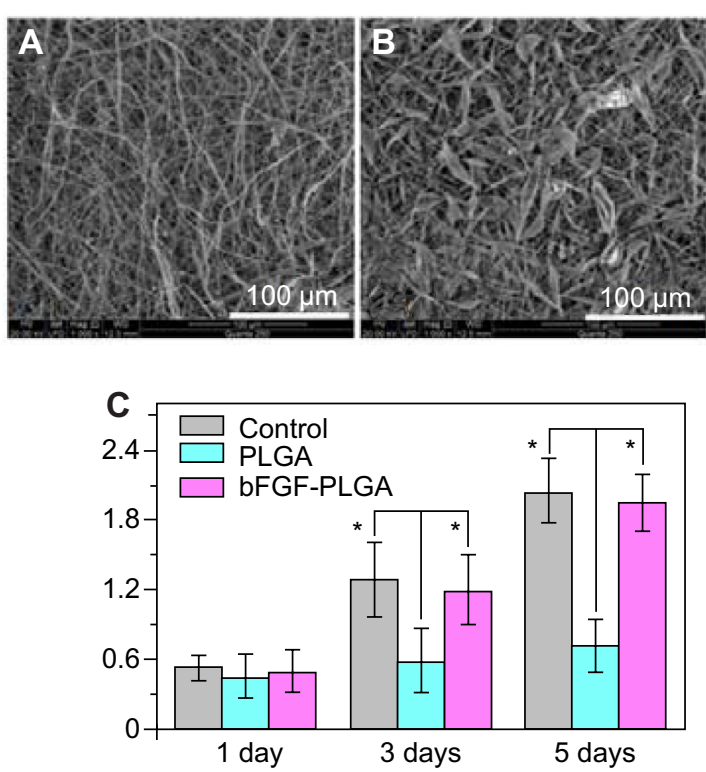

Figure 4 SEM images of HDF growth on the electrospun PLGA.

Notes: (A) and bFGF-PLGA; (B) fibrous membranes at 5 days after seeding; (C) CCK-8 assay of HDF proliferation on the electrospun fibrous membranes. *Represents $P<0.05$ compared to the corresponding PLGA group.

Abbreviations: bFGF, basic fibroblast growth factor; HDF, human dermal fibroblasts; PLGA, poly(lactide-co-glycolide); SEM, scanning electron microscopy.

the membrane fibers began to be absorbed, and fibroblasts were observed in the interstices of the fibers. Especially in the bFGF-PLGA group, these membranes were discernible and intimately associated with surrounding host tissues. Grafts exhibited variable regions of cellularity, and most of the cellular infiltrates were accounted for by fibroblasts infiltrating within and between the fascicles of the native fascia architecture. At 4 weeks, fibroblasts aligned irregularly with moderate cellularity and vascular formation at the interface between the membranes and host tissues. Cells aligned regularly and cellularity decreased from levels observed at 8 weeks. The fibrocartilaginous tissues with more parallel fibrous tissues were expressed at the tendon insertion site in the bFGF-PLGA group (Figure 5B). In comparison, the control group showed inferior healing capacity and regenerated more slowly at each time point.

\section{Metachromasia}

At 2 weeks after surgery, PLGA and bFGF-PLGA membranes significantly increased the area of glycosaminoglycan staining at the tendon-bone interface compared with the control group (354514 $\pm 18781 \mu \mathrm{m}^{2}$ for controls, $407041 \pm 16211 \mu \mathrm{m}^{2}$ for PLGA, and $430569 \pm 9109 \mu \mathrm{m}^{2}$ for bFGF-PLGA). The bFGF-PLGA group had a significantly greater total area of metachromasia compared with the PLGA group $(P=0.018)$. At 4 and 8 weeks after surgery, experimental groups had a larger total area of metachromasia compared with the

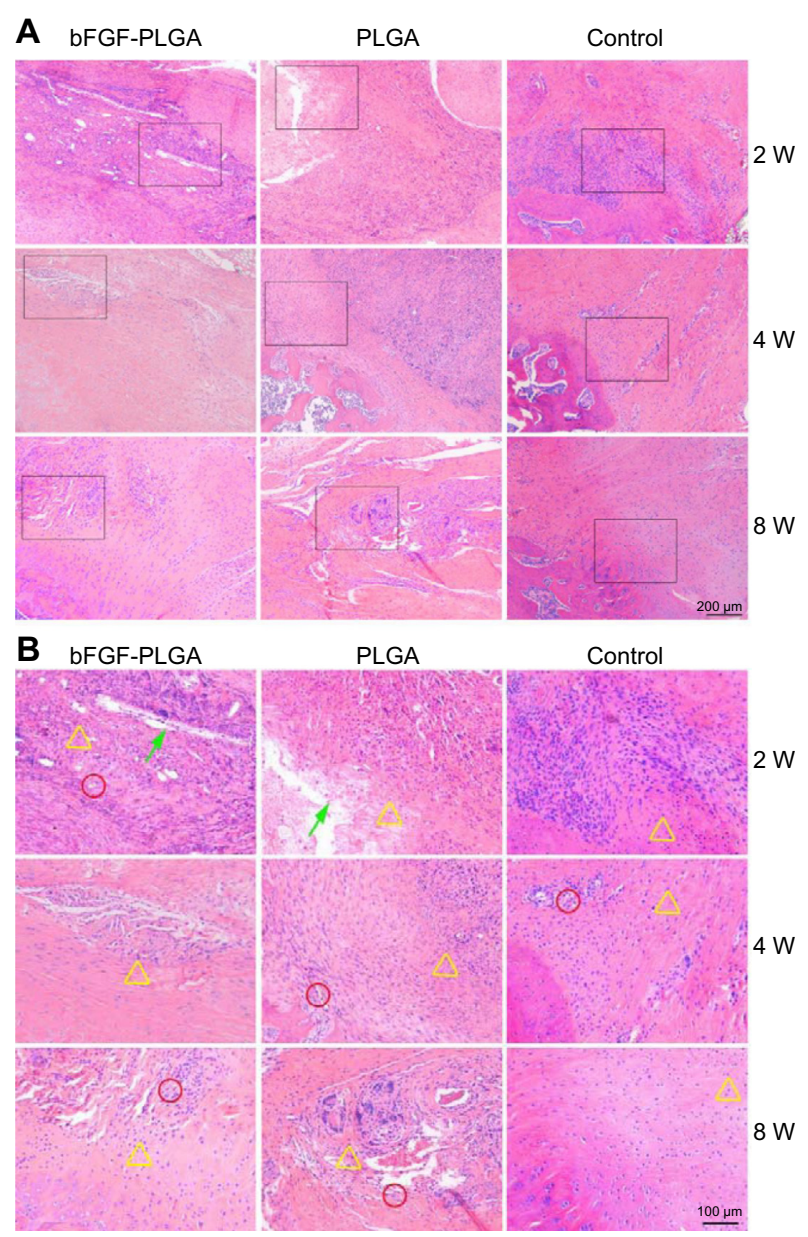

Figure 5 Representative hematoxylin and eosin-stained tissue sections.

Notes: (A) 100x and (B) 200x of the tendon insertion site at 2, 4, and 8 weeks postoperatively. The green arrow indicates degraded fibrous membrane, the yellow triangle indicates fibroblasts, and the red circle indicates microvessels.

Abbreviations: bFGF, basic fibroblast growth factor; PLGA, poly(lactide-co-glycolide); W, weeks.

control group $\left(402,175 \pm 14,574\right.$ and $409,823 \pm 14,374 \mu \mathrm{m}^{2}$ for controls, 444,898 $\pm 17,109$ and $472,893 \pm 12,230 \mu \mathrm{m}^{2}$ for PLGA, 459,096 $\pm 13,042$ and $488,769 \pm 11,389 \mu \mathrm{m}^{2}$ for bFGF-PLGA). There were no differences between the experimental groups (Figure 6). bFGF-PLGA exhibited the capacity to improve cartilage regeneration at the tendon-bone interface, especially at the early stage in which bFGF was initially released.

\section{Collagen organization}

At all three time points, the experimental groups had significantly improved collagen organization over the control group, as based on birefringence under polarized light at the healing enthesis $(18.4 \pm 0.6$ gray-scale units at 2 weeks, $29.7 \pm 0.9$ at 4 weeks, and $41.9 \pm 1.3$ at 8 weeks for controls; $20.5 \pm 0.9$ at 2 weeks, $31.4 \pm 0.7$ at 4 weeks, and $43.8 \pm 1.0$ at 8 weeks for PLGA; $22.6 \pm 0.7$ at 2 weeks, $32.7 \pm 0.8$ at 4 weeks, 
and $45.4 \pm 1.2 \mathrm{~g}$ at 8 weeks for bFGF-PLGA). Further, the collagen birefringence for the bFGF-PLGA groups was significantly greater than that for the PLGA group (Figure 7). These findings show that the bFGF-PLGA group improved collagen production.

\section{Biomechanical testing}

\section{Cross-sectional area of the healing enthesis}

At 2 weeks after surgery, there were no differences in the cross-sectional area of the healing enthesis between the control group and the experimental groups $\left(8.3 \pm 0.5 \mathrm{~mm}^{2}\right.$ for controls, $8.6 \pm 0.7 \mathrm{~mm}^{2}$ for PLGA, and $9.0 \pm 0.9 \mathrm{~mm}^{2}$ for bFGF-PLGA; $P=0.766$ and $P=0.218$, respectively).
However, at 4 weeks after surgery, there were significant differences in the area of the healing enthesis between the control group and the experimental groups $\left(14.0 \pm 1.5 \mathrm{~mm}^{2}\right.$ for controls, $15.7 \pm 1.0 \mathrm{~mm}^{2}$ for PLGA, and $16.1 \pm 1.1 \mathrm{~mm}^{2}$ for bFGF-PLGA; $P=0.037$ and $P=0.006$, respectively). There was no difference between the experimental groups. At 8 weeks, there were significant differences between the control group and the experimental groups (16.4 \pm 0.6 $\mathrm{mm}^{2}$ for controls, $17.8 \pm 0.9 \mathrm{~mm}^{2}$ for PLGA, and $18.4 \pm 0.6$ $\mathrm{mm}^{2}$ for bFGF-PLGA; Figure 8A). Increases in the crosssectional area in the experimental groups were most likely due to the extra thickness of the implanted membrane after 4 weeks.
A

bFGF-PLGA

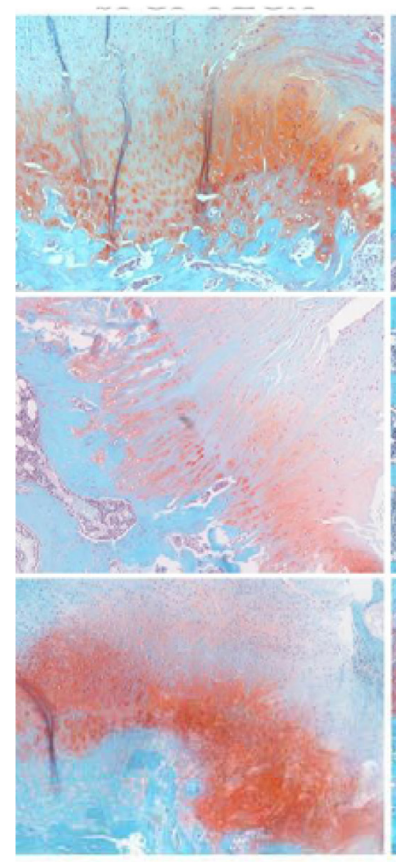

B

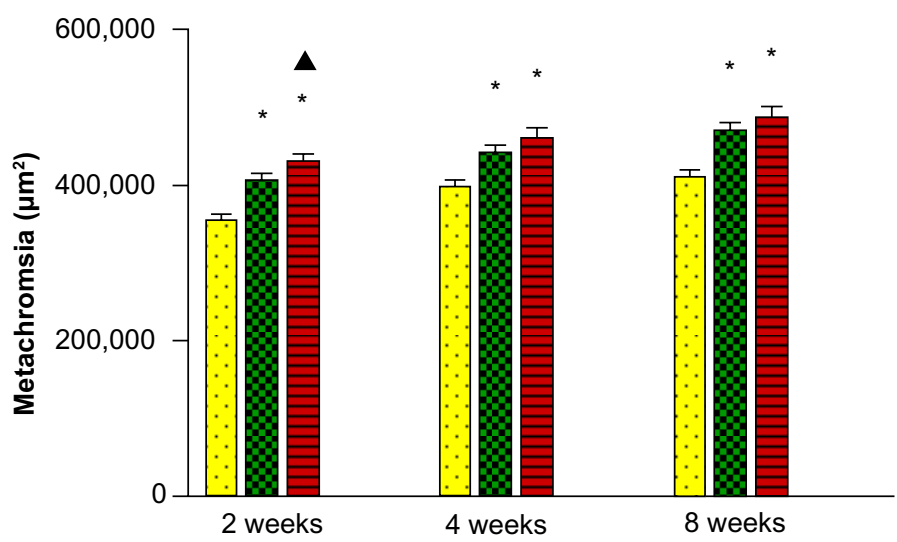

Control
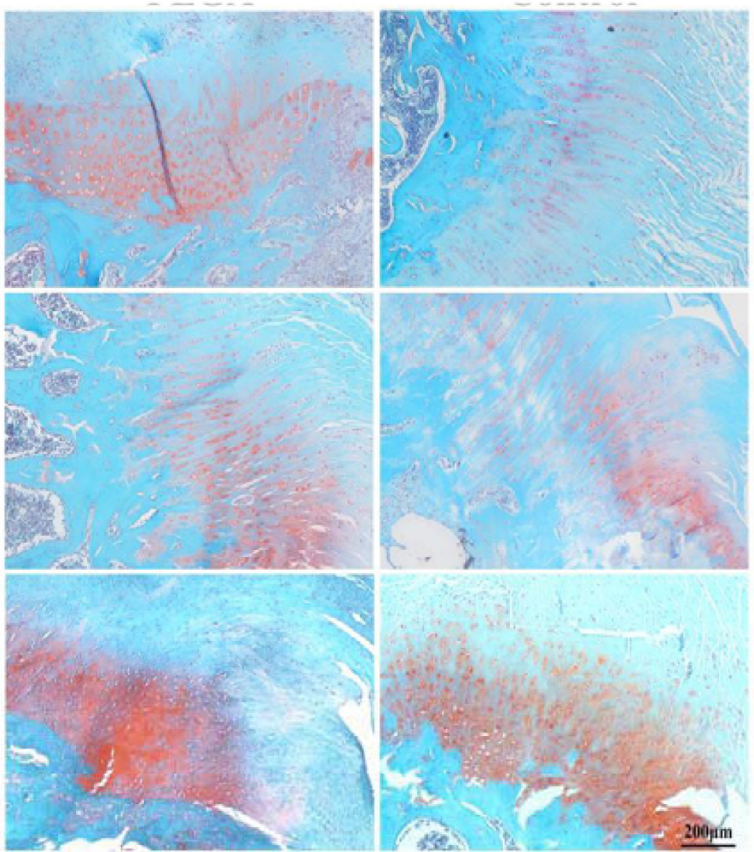

$2 \mathrm{~W}$

$4 \mathrm{~W}$

$8 \mathrm{~W}$

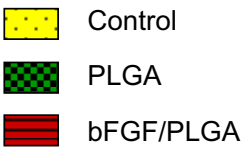

Figure 6 Representative histological images of cartilage at the insertion site (A) 100x, and area of cartilage present at the insertion site as determined by metachromasia with Safranin O-stained slides (B).

Notes: The results are shown as the mean $\pm S D$; ${ }^{*}$ represents $P<0.05$ versus control; ${ }^{\Delta}$ represents $P<0.05$ versus $P L G A ; n=8$ for each group.

Abbreviations: bFGF, basic fibroblast growth factor; PLGA, poly(lactide-co-glycolide); W, weeks; SD, standard deviation. 

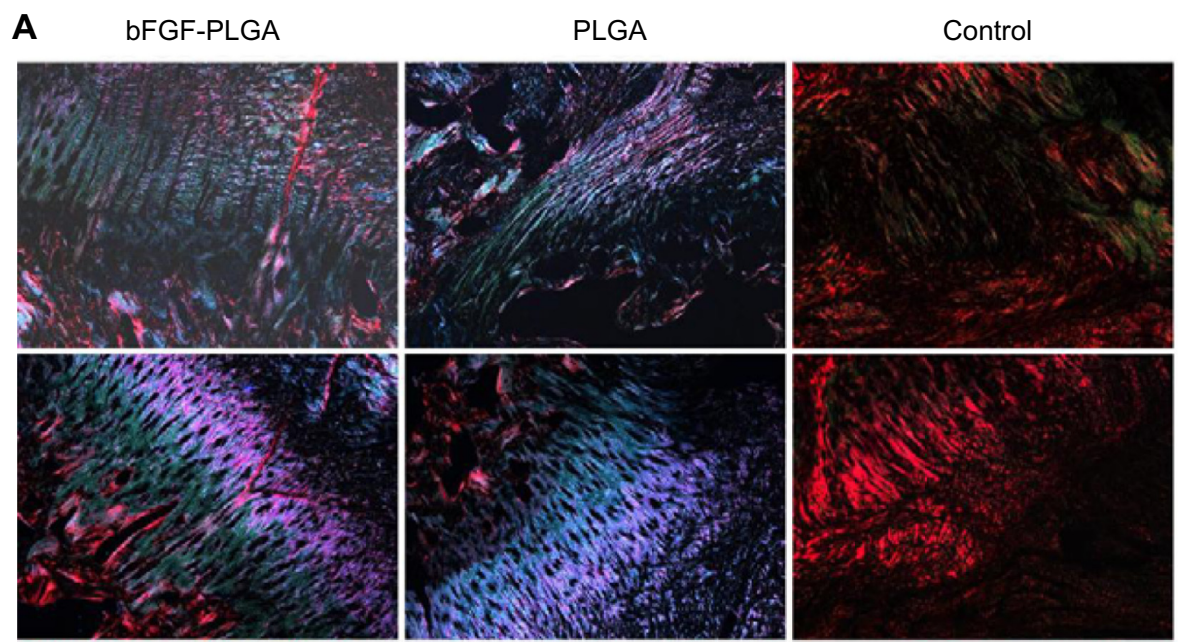

$4 \mathrm{~W}$
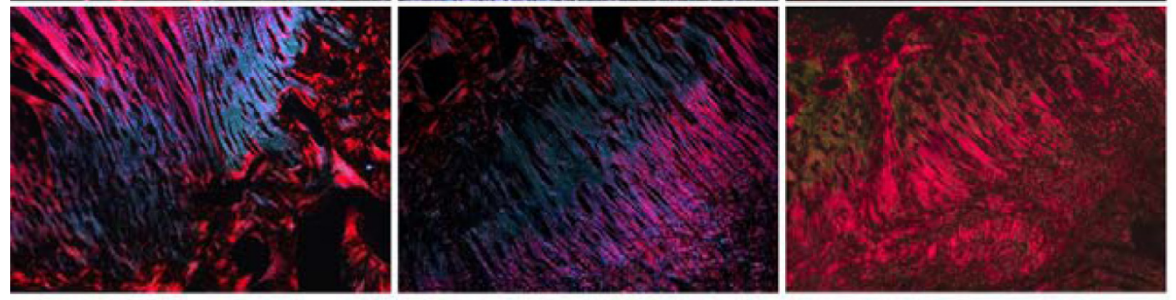

B

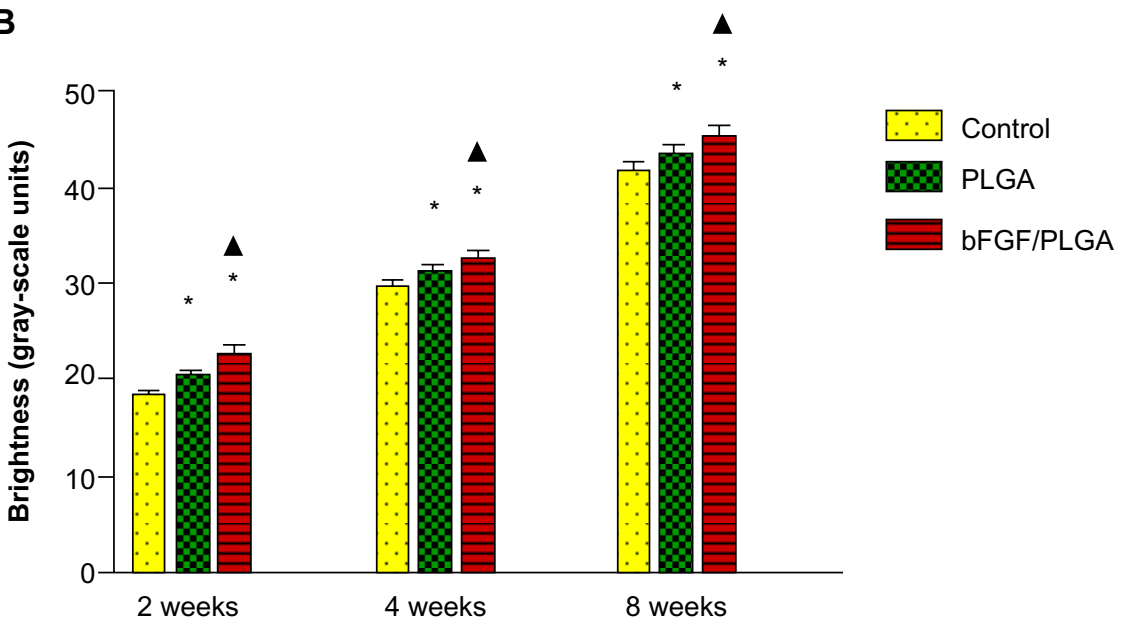

Figure 7 Representative Picro Sirius red-stained tissue sections of the healing enthesis (A) $100 \times$ and analysis of collagen birefringence (B). Notes: The results are shown as the mean \pm SD; *represents $P<0.05$ versus control; ${ }^{\mathbf{A}}$ represents $P<0.05$ versus $P L G A ; n=8$ for each group. Abbreviations: bFGF, basic fibroblast growth factor; PLGA, poly(lactide-co-glycolide); W, weeks; SD, standard deviation.

\section{Ultimate load-to-failure and stiffness}

At 2 weeks after surgery, there was no difference in the ultimate load-to-failure or stiffness of the supraspinatus tendon-bone construct between groups $(8.3 \pm 0.6 \mathrm{~N}$ and $5.4 \pm 0.6 \mathrm{~N} / \mathrm{mm}$ for controls, $8.7 \pm 0.9 \mathrm{~N}$ and $5.7 \pm 0.7 \mathrm{~N} /$ $\mathrm{mm}$ for PLGA, and $9.1 \pm 0.9 \mathrm{~N}$ and $6.1 \pm 0.6 \mathrm{~N} / \mathrm{mm}$ for bFGF-PLGA). However, at 4 weeks, there were significantly greater ultimate load-to-failure and stiffness of the supraspinatus tendon-bone construct in the experimental groups $(18.2 \pm 0.9 \mathrm{~N}$ and $7.9 \pm 0.8 \mathrm{~N} / \mathrm{mm}$ for controls, $20.7 \pm 1.6 \mathrm{~N}$ and $9.4 \pm 0.8 \mathrm{~N} / \mathrm{mm}$ for PLGA, and $21.4 \pm 1.3 \mathrm{~N}$ and $9.7 \pm 0.5 \mathrm{~N} / \mathrm{mm}$ for bFGF-PLGA). There was no difference between the experimental groups. At 8 weeks, there were significantly greater ultimate load-to-failure and stiffness of the supraspinatus tendon-bone construct in the repairs augmented with bFGF-PLGA compared with both PLGA and control groups $(27.1 \pm 1.2 \mathrm{~N}$ and $13.0 \pm 0.7 \mathrm{~N} / \mathrm{mm}$ for controls, $28.4 \pm 1.2 \mathrm{~N}$ and $13.7 \pm 0.7 \mathrm{~N} / \mathrm{mm}$ for PLGA, and $32.7 \pm 1.0 \mathrm{~N}$ and $14.9 \pm 0.3 \mathrm{~N} / \mathrm{mm}$ for bFGF-PLGA; Figure $8 \mathrm{~B}$ and $\mathrm{C}$ ). After 8 weeks, the mechanical strength of the supraspinatus tendon-bone construct augmented with bFGF-PLGA membrane increased noticeably. 
A

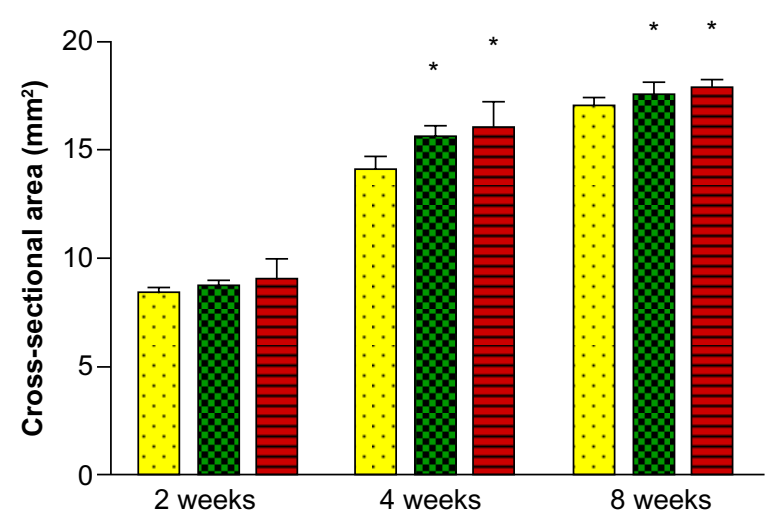

C

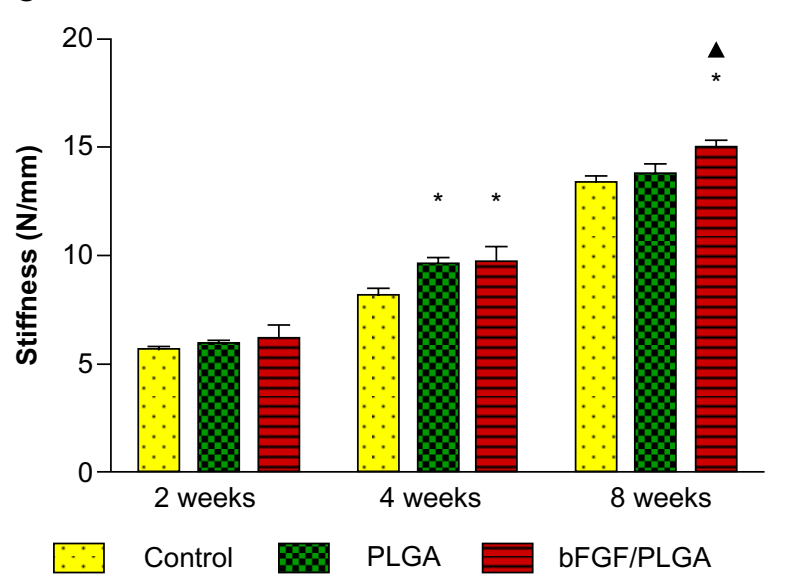

B

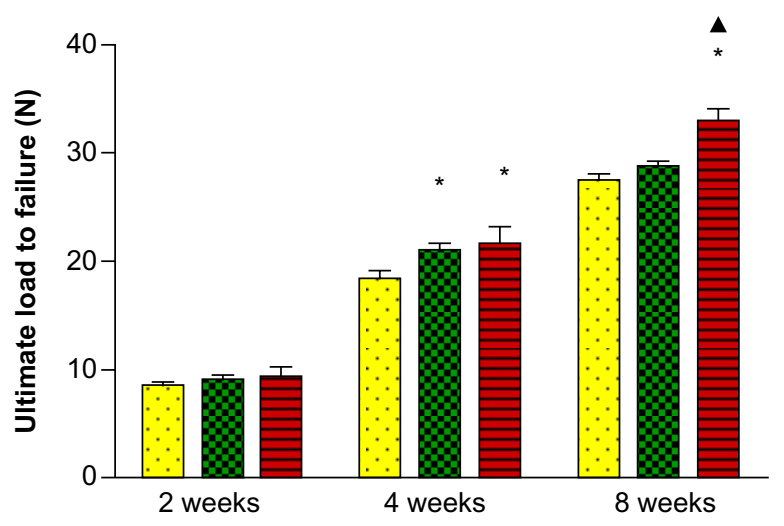

D $\quad$ A

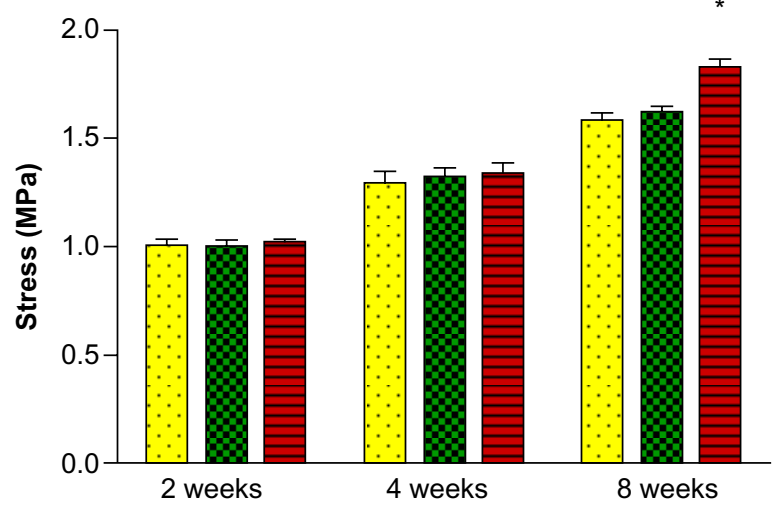

Figure 8 The cross-sectional area of the tendon at the insertion site (A), and biomechanical testing of ultimate load-to-failure (B), stiffness (C), and ultimate stress-to-failure (D). Notes: The results are shown as the mean \pm SD; *represents $P<0.05$ versus control; ${ }^{\star}$ represents $P<0.05$ versus $P L G A ; n=8$ for each group.

Abbreviations: bFGF, basic fibroblast growth factor; PLGA, poly(lactide-co-glycolide); W, weeks; SD, standard deviation.

\section{Ultimate stress of the healing enthesis}

At 2 and 4 weeks, there was no difference in the ultimate stress of the healing enthesis between groups $(1.00 \pm 0.02$ and $1.30 \pm 0.08 \mathrm{MPa}$ for controls, $1.00 \pm 0.02$ and $1.32 \pm 0.06 \mathrm{MPa}$ for PLGA, and $1.02 \pm 0.02$ and $1.33 \pm 0.05$ for bFGF-PLGA). At 8 weeks, there was significantly greater ultimate stress in the repairs augmented with bFGF-PLGA $(1.65 \pm 0.09 \mathrm{MPa}$ for controls, $1.62 \pm 0.03 \mathrm{MPa}$ for PLGA, $1.82 \pm 0.03$ for bFGFPLGA; Figure 8D). This was consistent with the results for ultimate load-to-failure and stiffness.

\section{Discussion}

The histopathology of chronic RC degeneration includes loss of cellularity, thinning, and disorganization of tendon fibers, increased granulation tissue, and fibrocartilaginous changes. ${ }^{31}$ This explains the relatively high percentage of patients with failure of healing or recurrent RCTs after repair. The pathogenesis results from mechanical and biological factors. Given the inadequate healing response, biological methods offer the possibility to augment RC repair and may be critical to improving clinical outcomes. ${ }^{28}$ However, acute RCT models, which most prior studies used for evaluation, did not reflect the degenerative, age-related RCTs commonly seen in humans who tend to present with intrinsic degenerative changes in the torn RC tendon. According to Buchmann's approach, ${ }^{25,26}$ we established a chronic rat RCT model in this study. Buchmann's team demonstrated that chronic human RCT could be imitated in the rat model, in which tendon degeneration, inflammation, and muscle atrophy combined with a persisting defect at 3 weeks after detachment were comparable to chronic tendon tears in humans.

In this study, we tested the hypothesis that treatment with bFGF-PLGA membrane can promote fibrocartilage formation, improve collagen organization, and strengthen the healing enthesis compared with repair alone in a chronic RCT model. We observed that the PLGA membranes showed a biocompatible host tissue response at the tendon repair sites. PLGA electrospun fibrous membrane is a kind 
of hydrophobic material, on which cell adhesion was poor in vitro. It did not promote cell proliferation at the early stage. While in vivo, the implanted membranes were wrapped by surrounding tissues and body fluid, which might change the material's characteristics and improve their biocompatibility. The electrospun nanofibers showed their ability to aid in cell attachment and proliferation, which made them undergo remodeling faster compared with those scaffolds derived from dermis. BFGF-loaded PLGA electrospun fibrous membranes with a core-sheath structure increased the encapsulation efficiency, retarded the initial bFGF burst release, and maintained bFGF bioactivity during the first 3 weeks. In the subsequent time period, proliferated cells secreted extracellular matrix and sustained $\mathrm{RC}$ tissue repair. Further, the PLGA membrane also exhibited excellent biodegradability. By 8 weeks after repairs, the PLGA membranes were replaced completely.

Local application of the PLGA membrane is associated with improvements in fibrocartilage and collagen organization at the healing enthesis compared with $\mathrm{RC}$ repair only at each time point, which underscores the importance of this material as a provisional matrix in the phase of tendonbone healing. Moreover, there was significantly improved collagen organization with bFGF-PLGA at all three time points, indicating that the bFGF-loaded PLGA membranes prolonged the $\mathrm{bFGF}$ action time and led to the formation of a more mature healing enthesis compared with PLGA alone or with repair only.

Biomechanical testing is critical to evaluate whether implantation of scaffolds can improve the biomechanical properties of the repair constructs. Biomechanical testing results determine the feasibility of augmentation with scaffolds. Several recent investigations showed improvements or no detrimental effects on mechanical properties using polymer scaffolds ${ }^{32,33}$ and heparin/fibrin-based systems. ${ }^{34}$ In this study, local application of the PLGA membrane did result in greater ultimate load-to-failure and stiffness at 4 weeks. Additionally, after 8 weeks, mechanical strength of the supraspinatus tendon-bone construct augmented with bFGF-PLGA membrane increased remarkably. Increases in the cross-sectional area of the experimental groups after 4 weeks were most likely caused by the extra thickness of the implanted membrane, which led to reductions of ultimate stress at any time point.

Synthetic scaffolds and matrices have been used in previous studies and have shown improvements in mechanical properties. Taylor et al used PLGA scaffolds in an overlay fashion to augment rat supraspinatus detachment and observed a dramatic increase in elastic modulus at 8 weeks with the scaffold compared with non-scaffold repairs. ${ }^{33}$ Manning et al used a heparin/fibrin-based gel as an overlay to deliver transforming growth factor-b3 (TGF-b3) to rat supraspinatus injury and repair. ${ }^{34}$ Inclusion of TGF-b3 in the scaffold improved mechanical properties, but scaffolds without TGF-b3 did not improve compared with repair-only controls. In multiple investigations, porcine small intestine submucosa has been used to augment repair, but these results showed no change compared with repair-only controls, nor were there improvements in mechanical properties over time after repair and implantation. ${ }^{35-37}$ Beason et al used overlaid scaffolds with and without fiber aligned to augment supraspinatus repair in rats and observed no significant differences in structural properties between the groups. ${ }^{38}$ All these studies are consistent with the present findings of no detrimental changes in structural properties caused by scaffold implantation. Our study, however, represents the first implementation of bFGF-loaded electrospun fibrous scaffolds followed by biomechanical and histologic evaluation. All of our results demonstrated meaningful improvement by combination therapy with bFGF and PLGA electrospun fibrous membranes in chronic RCT models.

Several limitations exist in this study. First, the RC repair used in this model differs from that used in humans. However, the relevance of this animal model has been established in the literature. ${ }^{27,39-42}$ Second, the single load-to-failure biomechanical test does not replicate the clinical setting, which is more consistent with a cyclic load application. ${ }^{17}$ Finally, although the byproducts of the PLGA scaffold are natural metabolites, the degradation products of synthetic polymers can cause inflammatory reactions when they accumulate at the implantation site. ${ }^{43-45}$ In this study, no inflammatory reactions were observed by 8 weeks postoperatively, but long-term follow-up is required to confirm these results. However, our study offers a novel biologic approach to augment RCT repairs with electrospun fibrous scaffolds loaded with growth factors.

\section{Conclusion}

Local application of bFGF-PLGA fibrous membranes to the healing tendon-bone interface after $\mathrm{RC}$ repair in a rat chronic RCT model was found to strengthen the healing enthesis, increase the area of fibrocartilage, and improve collagen organization compared with repair alone. RC regeneration using bFGF-PLGA is a promising treatment for RCT. Additional studies may also assess the effects of other growth factors such as platelet-derived growth factor, 
vascular endothelial growth factor, and TGF. Augmentation of the repair combined with concurrent drug delivery in this manner could enhance long-term healing after RC injury and repair and may eventually lead to improvement of clinical surgical outcome by enhancing tissue regeneration.

\section{Acknowledgment}

This work was supported by the National Natural Science Foundation of China (51373112, 51003058, and $31370945)$.

\section{Disclosure}

The authors report no conflicts of interest in this work.

\section{References}

1. Domb BG, Glousman RE, Brooks A, Hansen M, Lee TQ, ElAttrache NS. High-tension double-row footprint repair compared with reducedtension single-row repair for massive rotator cuff tears. J Bone Joint Surg Am. 2008;90 Suppl 4:35-39.

2. Nelson CO, Sileo MJ, Grossman MG, Serra-Hsu F. Single-row modified mason-allen versus double-row arthroscopic rotator cuff repair: a biomechanical and surface area comparison. Arthroscopy. 2008;24(8): 941-948.

3. Ozbaydar M, Elhassan B, Esenyel C, et al. A comparison of singleversus double-row suture anchor techniques in a simulated repair of the rotator cuff: an experimental study in rabbits. J Bone Joint Surg Br. 2008;90(10): 1386-1391.

4. Pietschmann MF, Froehlich V, Ficklscherer A, Wegener B, Jansson V, Muller PE. Biomechanical testing of a new knotless suture anchor compared with established anchors for rotator cuff repair. J Shoulder Elbow Surg. 2008;17(4):642-646.

5. Tocci SL, Tashjian RZ, Leventhal E, Spenciner DB, Green A, Fleming BC. Biomechanical comparison of single-row arthroscopic rotator cuff repair technique versus transosseous repair technique. J Shoulder Elbow Surg. 2008;17(5):808-814.

6. Oh JH, Kim SH, Ji HM, Jo KH, Bin SW, Gong HS. Prognostic factors affecting anatomic outcome of rotator cuff repair and correlation with functional outcome. Arthroscopy. 2009;25(1):30-39.

7. Ide J, Kikukawa K, Hirose J, et al. The effect of a local application of fibroblast growth factor-2 on tendon-to-bone remodeling in rats with acute injury and repair of the supraspinatus tendon. J Shoulder Elbow Surg. 2009;18(3):391-398.

8. Dines JS, Weber L, Razzano P, et al. The effect of growth differentiation factor-5-coated sutures on tendon repair in a rat model. J Shoulder Elbow Surg. 2007;16(Supp1 5):S215-S221.

9. Pelinkovic D, Lee JY, Engelhardt M, et al. Muscle cell-mediated gene delivery to the rotator cuff. Tissue Eng. 2003;9(1):143-151.

10. Thomopoulos S, Harwood FL, Silva MJ, Amiel D, Gelberman RH. Effect of several growth factors on canine flexor tendon fibroblast proliferation and collagen synthesis in vitro. J Hand Surg. 2005;30(3):441-447.

11. Takahasih S, Nakajima M, Kobayashi M, et al. Effect of recombinant basic fibroblast growth factor (bFGF) on fibroblast-like cells from human rotator cuff tendon. Tohoku J Exp Med. 2002;198(4):207-214.

12. Chang J, Most D, Thunder R, Mehrara B, Longaker MT, Lineaweaver WC. Molecular studies in flexor tendon wound healing: the role of basic fibroblast growth factor gene expression. J Hand Surg. 1998;23(6):1052-1058.

13. Khan U, Occleston NL, Khaw PT, McGrouther DA. Differences in proliferative rate and collagen lattice contraction between endotenon and synovial fibroblasts. J Hand Surg. 1998;23(2):266-273.
14. Kobayashi M, Itoi E, Minagawa H, et al. Expression of growth factors in the early phase of supraspinatus tendon healing in rabbits. J Shoulder Elbow Surg. 2006;15(3):371-377.

15. Wurgler-Hauri CC, Dourte LM, Baradet TC, Williams GR, Soslowsky LJ. Temporal expression of 8 growth factors in tendon-to-bone healing in a rat supraspinatus model. J Shoulder Elbow Surg. 2007;16(Suppl 5):S198-S203.

16. Tang JB, Cao Y, Zhu B, Xin KQ, Wang XT, Liu PY. Adeno-associated virus-2-mediated bFGF gene transfer to digital flexor tendons significantly increases healing strength. An in vivo study. J Bone Joint Surg Am. 2008;90(5):1078-1089.

17. Ide J, Kikukawa K, Hirose J, Iyama K, Sakamoto H, Mizuta H. The effects of fibroblast growth factor-2 on rotator cuff reconstruction with acellular dermal matrix grafts. Arthroscopy. 2009;25(6):608-616.

18. Derwin KA, Badylak SF, Steinmann SP, Iannotti JP. Extracellular matrix scaffold devices for rotator cuff repair. J Shoulder Elbow Surg. 2010;19(3):467-476.

19. Zamani F, Amani-Tehran M, Latifi M, Shokrgozar MA. The influence of surface nanoroughness of electrospun PLGA nanofibrous scaffold on nerve cell adhesion and proliferation. J Mater Sci Mater Med. 2013;24(6):1551-1560.

20. $\mathrm{Hu} \mathrm{C}$, Cui W. Hierarchical structure of electrospun composite fibers for long-term controlled drug release carriers. Adv Healthc Mat. 2012;1(6):809-814.

21. Cui W, Cheng L, Hu C, Li H, Zhang Y, Chang J. Electrospun poly(Llactide) fiber with ginsenoside $\mathrm{rg} 3$ for inhibiting scar hyperplasia of skin. PloS one. 2013;8(7):e68771.

22. Sahoo S, Ang LT, Goh JC, Toh SL. Growth factor delivery through electrospun nanofibers in scaffolds for tissue engineering applications. J Biomed Mater Res A. 2010;93(4):1539-1550.

23. Yang Y, Xia T, Zhi W, et al. Promotion of skin regeneration in diabetic rats by electrospun core-sheath fibers loaded with basic fibroblast growth factor. Biomaterials. 2011;32(18):4243-4254.

24. Liu S, Qin M, Hu C, et al. Tendon healing and anti-adhesion properties of electrospun fibrous membranes containing bFGF loaded nanoparticles. Biomaterials. 2013;34(19):4690-4701.

25. Buchmann S, Sandmann GH, Walz L, et al. Refixation of the supraspinatus tendon in a rat model - influence of continuous growth factor application on tendon structure. J Orthop Res. 2013;31(2): 300-305.

26. Buchmann S, Walz L, Sandmann GH, et al. Rotator cuff changes in a full thickness tear rat model: verification of the optimal time interval until reconstruction for comparison to the healing process of chronic lesions in humans. Arch Orthop Trauma Surg. 2011;131(3):429-435.

27. Thomopoulos S, Hattersley G, Rosen V, et al. The localized expression of extracellular matrix components in healing tendon insertion sites: an in situ hybridization study. J Orthop Res. 2002;20(3):454-463.

28. Kovacevic D, Fox AJ, Bedi A, et al. Calcium-phosphate matrix with or without TGF-beta3 improves tendon-bone healing after rotator cuff repair. Am J Sports Med. 2011;39(4):811-819.

29. Bedi A, Fox AJ, Kovacevic D, Deng XH, Warren RF, Rodeo SA. Doxycycline-mediated inhibition of matrix metalloproteinases improves healing after rotator cuff repair. Am J Sports Med. 2010;38(2): 308-317.

30. Gulotta LV, Kovacevic D, Montgomery S, Ehteshami JR, Packer JD, Rodeo SA. Stem cells genetically modified with the developmental gene MT1-MMP improve regeneration of the supraspinatus tendon-to-bone insertion site. Am J Sports Med. 2010;38(7):1429-1437.

31. Bedi A, Maak T, Walsh C, et al. Cytokines in rotator cuff degeneration and repair. J Shoulder Elbow Surg. 2012;21(2):218-227.

32. Hee CK, Dines JS, Dines DM, et al. Augmentation of a rotator cuff suture repair using rhPDGF-BB and a type I bovine collagen matrix in an ovine model. Am J Sports Med. 2011;39(8):1630-1639.

33. Taylor ED, Nair LS, Nukavarapu SP, McLaughlin S, Laurencin CT. Novel nanostructured scaffolds as therapeutic replacement options for rotator cuff disease. J Bone Joint Surg Am. 2010;92 Suppl 2:170-179. 
34. Manning CN, Kim HM, Sakiyama-Elbert S, Galatz LM, Havlioglu N, Thomopoulos S. Sustained delivery of transforming growth factor beta three enhances tendon-to-bone healing in a rat model. J Orthop Res. 2011;29(7):1099-1105.

35. Nicholson GP, Breur GJ, Van Sickle D, Yao JQ, Kim J, Blanchard CR. Evaluation of a cross-linked acellular porcine dermal patch for rotator cuff repair augmentation in an ovine model. J Shoulder Elbow Surg. 2007;16(Suppl 5):S184-S190.

36. Perry SM, Gupta RR, Van Kleunen J, Ramsey ML, Soslowsky LJ, Glaser DL. Use of small intestine submucosa in a rat model of acute and chronic rotator cuff tear. J Shoulder Elbow Surg. 2007;16(Suppl 5): S179-S183.

37. Zalavras CG, Gardocki R, Huang E, Stevanovic M, Hedman T, Tibone J. Reconstruction of large rotator cuff tendon defects with porcine small intestinal submucosa in an animal model. J Shoulder Elbow Surg. 2006;15(2):224-231

38. Beason DP, Connizzo BK, Dourte LM, et al. Fiber-aligned polymer scaffolds for rotator cuff repair in a rat model. J Shoulder Elbow Surg. 2012;21(2):245-250.

39. Soslowsky LJ, Carpenter JE, DeBano CM, Banerji I, Moalli MR. Development and use of an animal model for investigations on rotator cuff disease. J Shoulder Elbow Surg. 1996;5(5):383-392.
40. Carpenter JE, Thomopoulos S, Flanagan CL, DeBano CM, Soslowsky LJ. Rotator cuff defect healing: a biomechanical and histologic analysis in an animal model. J Shoulder Elbow Surg. 1998;7(6):599-605.

41. Soslowsky LJ, Thomopoulos S, Tun S, et al. Neer Award 1999. Overuse activity injures the supraspinatus tendon in an animal model: a histologic and biomechanical study. J Shoulder Elbow Surg. 2000;9(2):79-84.

42. Thomopoulos S, Soslowsky LJ, Flanagan CL, et al. The effect of fibrin clot on healing rat supraspinatus tendon defects. J Shoulder Elbow Surg. 2002;11(3):239-247.

43. Bostman O, Pihlajamaki H. Clinical biocompatibility of biodegradable orthopaedic implants for internal fixation: a review. Biomaterials. 2000;21(24):2615-2621.

44. Cao D, Liu W, Wei X, Xu F, Cui L, Cao Y. In vitro tendon engineering with avian tenocytes and polyglycolic acids: a preliminary report Tissue Eng. 2006;12(5):1369-1377.

45. Inui A, Kokubu T, Mifune Y, et al. Regeneration of rotator cuff tear using electrospun poly(d,1-lactide-co-glycolide) scaffolds in a rabbit model. Arthroscopy. 2012;28(12):1790-1799.
International Journal of Nanomedicine

\section{Publish your work in this journal}

The International Journal of Nanomedicine is an international, peerreviewed journal focusing on the application of nanotechnology in diagnostics, therapeutics, and drug delivery systems throughou the biomedical field. This journal is indexed on PubMed Central,

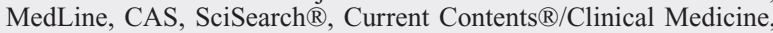

\section{Dovepress}

Journal Citation Reports/Science Edition, EMBase, Scopus and the Elsevier Bibliographic databases. The manuscript management system is completely online and includes a very quick and fair peer-review system, which is all easy to use. Visit http://www.dovepress.com/ testimonials.php to read real quotes from published authors. 